\title{
Cost-Effectiveness Analysis of Neoadjuvant Chemotherapy with Zoledronic Acid for HER2-Negative Breast Cancer in Japan: The JONIE1 Study
}

\author{
Kyoko Nakazawa ${ }^{1,2 *}$, Shota Saito ${ }^{3}$, Masayuki Nagahashi4, \\ Akimitsu Yamada ${ }^{5}$, Akira Toyama², Kouhei Akazawa ${ }^{6}$ \\ ${ }^{1}$ Department of Medical Informatics and Statistics, Niigata University Graduate School of Medical and Dental Sciences, Niigata, Japan \\ ${ }^{2}$ Department of Pharmacy, Niigata University Medical and Dental Hospital, Niigata, Japan \\ ${ }^{3}$ Department of Health Informatics, Niigata University of Health and Welfare, Niigata, Japan \\ ${ }^{4}$ Division of Digestive and General Surgery, Niigata University Graduate School of Medical and Dental Sciences, Niigata, Japan \\ ${ }^{5}$ Department of Gastroenterological Surgery, Yokohama City University School of Medicine, Kanagawa, Japan \\ ${ }^{6}$ Department of Medical Informatics, Niigata University Medical and Dental Hospital, Niigata, Japan \\ Email: *nakakyo-nii@umin.ac.jp
}

How to cite this paper: Nakazawa, K., Saito, S., Nagahashi, M., Yamada, A., Toyama, A. and Akazawa, K. (2019) Cost-Effectiveness Analysis of Neoadjuvant Chemotherapy with Zoledronic Acid for HER2-Negative Breast Cancer in Japan: The JONIE1 Study. Health, 11, 1017-1027.

https://doi.org/10.4236/health.2019.118080

Received: July 11, 2019

Accepted: August 10, 2019

Published: August 13, 2019

Copyright $\odot 2019$ by author(s) and Scientific Research Publishing Inc. This work is licensed under the Creative Commons Attribution International License (CC BY 4.0).

http://creativecommons.org/licenses/by/4.0/

(c) (i) Open Access

\begin{abstract}
Objective: Zoledronic acid (ZOL) is a nitrogen-containing bisphosphonate that induces osteoclast apoptosis and inhibits bone resorption. Adding ZOL to neoadjuvant chemotherapy has been shown to have potential anticancer benefits in women with HER2-negative breast cancer. The objective of the present study was to investigate ZOL's cost-effectiveness from the perspective of health care payers in Japan. Methods: A Markov model was developed to evaluate the costs and effectiveness associated with $\mathrm{ZOL}+$ chemotherapy (CTZ) and chemotherapy (CT) alone over a 10-year time horizon. Monthly transition probabilities were estimated according to JONIE1 (Japan Organization of Neoadjuvant Innovative Expert) Study data and an extrapolated Weibull model. Health outcomes were measured in quality-adjusted life years (QALYs). Costs were calculated using year-2018 Japanese yen (JPY) (1.00 US dollars $(\mathrm{USD})=110.4 \mathrm{JPY})$. Model robustness was addressed through one-way and probabilistic sensitivity analysis. The costs and QALYs were discounted at a rate of $2 \%$ per year. Results: In the base case, the use of CTZ was associated with a gain of 3.94 QALYs. The incremental cost per QALY of the CTZ gain was 681,056.1 JPY (6168.99 USD) per QALY. Conclusion: It is convincing that neoadjuvant CTZ for patients with breast cancer would be expected to have statistically significant clinical efficacy. Addition of ZOL to CT might be a cost-effective option compared with $\mathrm{CT}$ alone.
\end{abstract}




\section{Keywords}

Cost-Effectiveness, Incremental Cost-Effectiveness Ratio (ICER), Quality-Adjusted

Life Year (QALY), Chemotherapy, HER2-Negative Breast Cancer

\section{Introduction}

Breast cancer is the most commonly diagnosed malignancy and the leading cause of cancer-related mortality among women worldwide [1]. In Japanese women, breast cancer is the most common cancer. It accounts for more than $50 \%$ of women diagnosed with cancer at age 40 - 60 years [2].

Breast cancer has been divided into different subtypes defined according to the cancer cells' receptors. Two hormone receptors (HRs) found on cancer cells are the estrogen receptor (ER) and progesterone receptor (PgR). More than two-thirds of breast cancer cases are classified as $\mathrm{HR}$-positive $(\mathrm{HR}+)$, and the proportion of $\mathrm{HR}+$ patients is high in Japanese women. Of all breast cancer cases, $15 \%$ - $25 \%$ show expression of the HER2 gene and protein, and those cases have poor prognosis [3]. The triple-negative type of breast cancer is a poor prognosis because it lacks not only the HER2 protein but also two HRs.

Treatments for breast cancer include drug therapy, surgery, and radiation therapy. Drug therapy for breast cancer consists of adjuvant chemotherapy (CT) to prevent recurrences. According to the stage of breast cancer, neoadjuvant CT may be conducted [3].

Bisphosphonates have a high affinity for hydroxyapatite on the bone surface and inhibit osteoclastic bone resorption. Zoledronic acid (ZOL) is a nitrogen-containing bisphosphonate that powerfully suppresses bone resorption for preventing skeletal morbidity secondary to metastatic bone cancer [4]. In in vitro experimental systems, ZOL reportedly enhances apoptotic effects when used concomitantly with anticancer agents. Antitumor effects of ZOL in combination with antitumor agents in vivo have also been reported [5], and ZOL inhibits farnesyl diphosphate synthase within the mevalonate pathway [4].

Therefore, we conducted the phase 2 randomized Japan Organization of Neoadjuvant Innovative Experts 1 (JONIE1) study to examine the additional effects of ZOL to CT [6]. This study reported 3 years of pathologic complete response (pCR), disease-free survival (DFS) rate, and overall survival (OS) rate data. Exploratory subset analysis of our previous study showed that postmenopausal women and patients with breast cancer of triple-negative type showed the most promising benefits from treatment with additional ZOL [7].

Another study, ClinicalTrials.gov NCT00242203, reported that ZOL administered with CT may improve DFS and OS in women with ER-stage II/III breast cancer. ZOL was administered every 3 weeks for 1 year (total 17 doses), commencing with the first dose of CT [8]. Thus, ZOL effects OS and DFS when used for neoadjuvant CT. 
However, we need to estimate the value and cost-effectiveness of this clinical efficacy, as it is essential to evaluate the effects of new drugs and specific interventions on prognosis. We conducted a cost-effectiveness analysis to compare CTZ with commonly used CT alone in treatment of women with HR+/HER2-negative breast cancer.

\section{Material and Methods}

\subsection{Target Population}

Our study used data from the JONIE1 Study [6] [7]. The study design and patient eligibility were reported previously. Briefly, 188 breast cancer women participated that included histologically proven invasive $\mathrm{HR}+$ or triple-negative type breast cancer of clinical stage IIA to IIIB, and randomly assigned to either the chemotherapy with CTZ group $(n=93)$ or the CT group $(n=95)$. The 3 -y DFS rate was $90.7 \%$ in the CTZ group, and $84.6 \%$ in CT group. Also, 6 of the 88 patients died in the CT-Z group, whereas 4 of the 92 patients died in the CT group [7].

CT group patients received four cycles of FEC100 (fluorouracil $500 \mathrm{mg} / \mathrm{m}^{2}$, epirubicin $100 \mathrm{mg} / \mathrm{m}^{2}$, and cyclophosphamide $500 \mathrm{mg} / \mathrm{m}^{2}$ ) administered by IV infusion every 3 weeks followed by 12 cycles of paclitaxel $80 \mathrm{mg} / \mathrm{m}^{2}$ by IV infusion once weekly.

CTZ group patients received ZOL $4 \mathrm{mg}$ was administered by IV infusion 4 times every 3 weeks during FEC100 administration and 3 times every 4 weeks during paclitaxel administration.

The protocol, including the documentation of informed consent and patient information, was approved by the independent ethics committee at each participating site. The study was performed in accordance with the International Conference on Harmonization guidelines concerning Good Clinical Practice and the Declaration of Helsinki. The study investigators provided an information form approved by each institutional review board to all patients before enrollment to explain the following, and obtain voluntary written informed consent to participate in the study from the patient.

\subsection{Model Overview}

A Markov model was developed to estimate the opportunity costs and the number of patients in each health state (Figure 1). The Markov model consisted of 4 health states: stable, progression and hormonal CT, progression and CT, and death. The analysis was conducted from the perspective of health care payers in Japan. The length of each Markov cycle was 1 month, and the time horizon was 10 years [9].

Patients received the initial treatment (i.e., FEC100 + paclitaxel \pm ZOL and surgery) in the stable state and discontinued the treatment when they transitioned to the progressive state. After disease progression, $\mathrm{HR}+$ patients received hormonal therapy, then a mix of CT regimens and palliative care. They received 


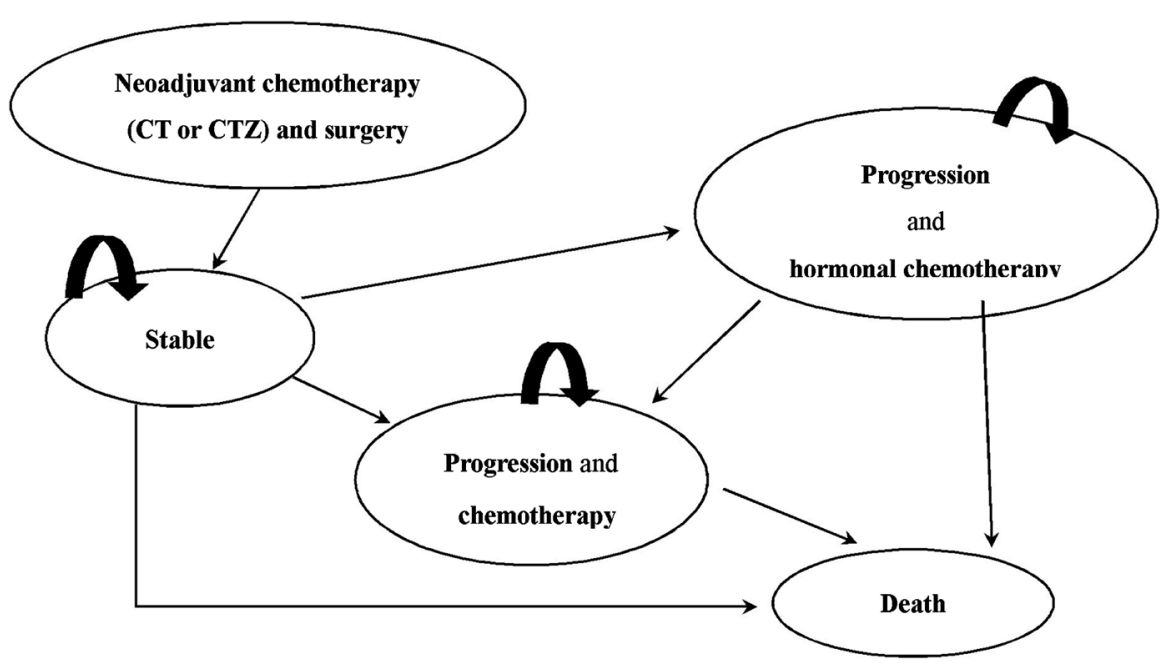

Figure 1. Markov model for cost-effectiveness analysis from beginning treatment to outcome.

hormonal therapy regimens for 18 months in the progression and hormonal CT state. Furthermore, patients in the disease progression and CT state received CT regimens and palliative care for an additional 6 months. HR-patients were moved to the CT state without transiting through the translated CT state.

Both breast cancer-related and non-breast cancer-related death were considered in the model.

\subsection{Estimate of Transition Probability}

To estimate the transition probability from the stable state to the progressive state, time to progression data were obtained from the JONIE1 Study. We decided the state of progression and hormonal CT or progression and CT based on the regimen.

The time horizon of 10 years was adopted to reflect the patients' limited remaining life span. Weibull curves were extrapolated to fit with Kaplan-Meier survival curves. The probability of transition was estimated according to the hazard levels of the survival models, and a Weibull model was used. These parameters were used to measure the probability of transition during cycle $t$, according to the formula

$$
P(t)=1-\exp \left[\lambda(t-1)^{\gamma}-\lambda t^{\gamma}\right][10]
$$

The scale $(\lambda)$ and shape $(\gamma)$ parameters were $\lambda=0.005, \gamma=1.43$ in CTZ and $\lambda$ $=0.0077, \gamma=1.56$ in CT.

The transition probability to death was extrapolated from the Weibull curves. The scale $(\lambda)$ and shape $(\gamma)$ parameters were $\lambda=0.035, \gamma=1.65$ in both CTZ and CT.

\subsection{Cost}

Because our study was conducted from the perspective of health care payers in Japan, only direct costs were considered in the model. In addition to drug and drug administration costs, patients also incurred monitoring costs during treatment and other related inpatient and outpatient costs. For example, some in- 
curred costs for bone metastasis management. Additional costs included drug, administration, and medical costs associated with each state and terminal care costs.

All cost inputs were obtained from the literature or publicly available data. Costs were calculated using year-2018 Japanese yen (JPY) (1.00 US dollars $(\mathrm{USD})=110.4 \mathrm{JPY})$ according to the social insurance reimbursement schedule and drug tariff of the fee-for-service system in Japan [11]. A breast cancer clinician was advised to estimate the costs of laboratory testing after surgery. We show the costs associated with each state (Table 1).

The cost of initial treatment $($ FEC100 + paclitaxel \pm ZOL $)$ was based on data from the patients enrolled in this study. The mean body surface area was $1.5 \mathrm{~m}^{2}$, and the number of vials of each drug was decided accordingly. If a drug had multiple package sizes, the price of the smallest package was used in the calculation. However, the largest package size for cyclophosphamide was used to account for the cost of preparative tools and medical service fees. Laboratory testing costs were calculated according to the protocol of the JONIE1 Study. Surgical costs were estimated based on the medical service fees of the Diagnosis Procedure Combination/Per-Diem Payment System in Japan. The postsurgical adjuvant therapy treatment was assumed to consist of radiation therapy and hormonal CT (tamoxifen $20 \mathrm{mg}$ daily).

The adjuvant CT treatment was tamoxifen (20 mg daily) and leuprorelin acetate (11.25 mg every 12 weeks). The subjects received hormonal CT regimens for 18 months. We decided the length of treatment based on the mean of cycles until progression from the JONIE1 Study.

Patients in the disease progression and CT state received a mix of CT regimens (Vinorelbine bitartrate $30 \mathrm{mg} / \mathrm{m}^{2}$ on day 1,8 times every 3 weeks) and palliative care for 6 months. The body surface area used was $1.5 \mathrm{~m}^{2}$ for both neoadjuvant CT and CT for progression. Then, they received outpatient CT. The model assumed that patients incurred one-time terminal care costs before death, an estimate of which was obtained from the opinions of breast cancer clinicians.

\subsection{Health-Related Utility}

A literature review was conducted to obtain health-related utilities for each state (stable, and progression and CT) [12] [13]. The health-related utility of the progressive and hormonal CT state was decided by a breast cancer clinician. Values for health-related quality of life, in which 1 year of life is multiplied by a utility factor between 0 (death) and 1 (perfect health), were taken from Miguel et al., who used the EQ-5D quality of life questionnaire to estimate utility scores in patients with breast cancer (Table 1).

\subsection{Cost-Effectiveness Analysis}

Model outputs included total costs and total effectiveness measured according to quality-adjusted life years (QALYs) associated with each treatment arm. QALYs 
Table 1. Key model inputs.

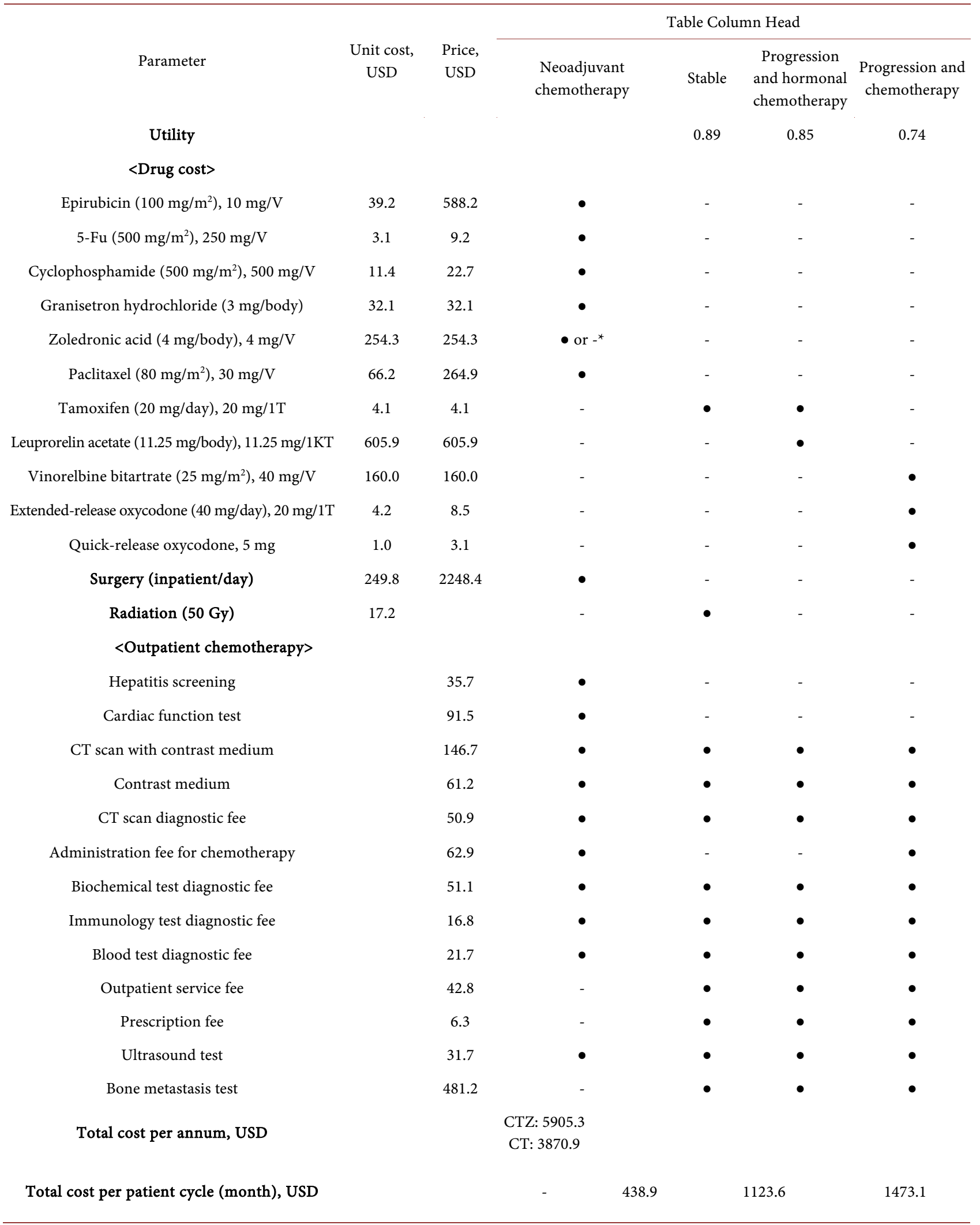

included; -: not included T: Tablet; V: Vial; KT: Kit; CT: chemotherapy; CTZ: Chemotherapy with Zoledronic Acid *: CT:-; CTZ: Sample of a Table footnote (Table footnote is dispensable). 
were estimated as the time spent in each state weighted by the utility value of each state. Incremental cost-effectiveness ratios (ICERs), measured as incremental costs per QALY gained, were estimated by comparing CTZ with CT. The costs and effectiveness outcomes were discounted at $2 \%$ annually. This discount value was based on the Guideline for the Economic Evaluation of Healthcare Technologies in Japan [14].

\subsection{Sensitivity Analysis}

One-way and multivariate probabilistic sensitivity analysis (PSA) were used to test the model's robustness. For these sensitivity analyses, we selected the parameters of the costs of the CTZ group (including those of neoadjuvant CT, surgery, and the progression and CT state), the utility value of the stable state, and the duration of the progression and hormonal CT state. One-way sensitivity analysis assessed the effects of varying key model parameters on the ICER. The costs of the CT state varied within a range of $25 \%$, and the duration of hormonal therapy varied by \pm 6 months because treatment details differed between individual patients. The utility value of the stable state varied within a range of $[-20 \%,+11 \%]$ considering adverse events from surgery or hormonal therapy.

A multivariate PSA was also conducted using a 10,000-iteration Monte Carlo simulation. PSA simultaneously varied multiple parameters that were randomly drawn from the prespecified distributions and estimated the ICERs. This included the PSA model input costs of the CTZ group (including those of neoadjuvant CT, surgery, and the progression and CT state), the utility value of the stable state, and the duration of the progression and hormonal CT state. A gamma distribution was used for each cost parameter, including that of neoadjuvant CT, surgery, CT state, and duration of hormonal therapy, and a beta distribution was used for the utility value of the stable state.

Additionally, a threshold analysis was performed to determine the cost-effectiveness price of ZOL when the willingness-to-pay was assumed to be $1,200,000$ JPY (10,869.6 USD).

All of the analyses were performed using TreeAge Pro software version 2018 (TreeAge, Williamstown, MA) and JMP ${ }^{\circledR} 14$ (SAS Institute Inc., Cary, NC, USA).

\section{Results}

\subsection{Base-Case Analysis}

The base-case analysis showed that the CTZ group was associated with additional total costs of 5,699,154 JPY (51,622.8 USD) for CT alone and 4,988,364 JPY $(45,184.5$ USD) total compared with the CT group. The QALYs gained in the CTZ and CT groups were 3.94 and 2.9, respectively, over 10 years. The ICER gained in a comparison of CTZ versus CT was 681,056.1 JPY (6168.99 USD).

\subsection{Sensitivity Analysis}

The one-way sensitivity analysis resulted in ICERs ranging 465,428.2 - 1,464,472 
JPY (4215.8 - 13,265.1 USD) per QALY (Table 2).

A PSA of 10,000 iterations indicated that the mean ICER value was 660,000 JPY (5928.3 USD) per QALY. At a willingness-to-pay threshold of 1,200,000 JPY (10,869.6 USD) per QALY, ZOL was considered cost-effective in $90 \%$ of the tested scenarios (Figure 2).

\section{Discussion}

ZOL is a nitrogen-containing bisphosphonate that powerfully suppresses bone resorption. It was clinically developed to prevent skeletal morbidity secondary to metastatic bone cancer. Previous studies have suggested a direct antitumor effect of ZOL and synergistic effects in combination with anticancer agents. Therefore, we planned a new trial to define the additional efficacy of ZOL to that of standard

Table 2. Table type styles (Table caption is indispensable).

\begin{tabular}{|c|c|c|c|c|}
\hline \multirow{2}{*}{ Table Head } & \multicolumn{3}{|c|}{ Value } & \\
\hline & base-case & sensi & analysis & \\
\hline \multirow{2}{*}{$\begin{array}{l}\text { Cost for CTZ group, including neoadjuvant } \\
\text { chemotherapy and surgery (USD) }\end{array}$} & 8153.7 & $+25 \%$ & $10,192.1$ & 8122.1 \\
\hline & & $-25 \%$ & 6115.3 & 4215.8 \\
\hline \multirow{2}{*}{ Utility value of stable state } & 0.89 & $+11 \%$ & 1 & 4710.2 \\
\hline & & $-20 \%$ & 0.7 & 13265.1 \\
\hline Duration of progression and hormonal & 18 & +6 & 24 & 6049.7 \\
\hline chemotherapy state (months) & & -6 & 12 & 6265.5 \\
\hline \multirow{2}{*}{$\begin{array}{l}\text { Cost of progression and chemotherapy state } \\
\text { (USD) }\end{array}$} & & $+25 \%$ & 1841.3 & 5856.2 \\
\hline & & $-25 \%$ & 1104.8 & 6481.8 \\
\hline
\end{tabular}

CT: Chemotherapy, CTZ: Chemotherapy with Zoledronic Acid, QALY: Quality-adjusted life years, ICER: Incremental cost-effectiveness ratios.

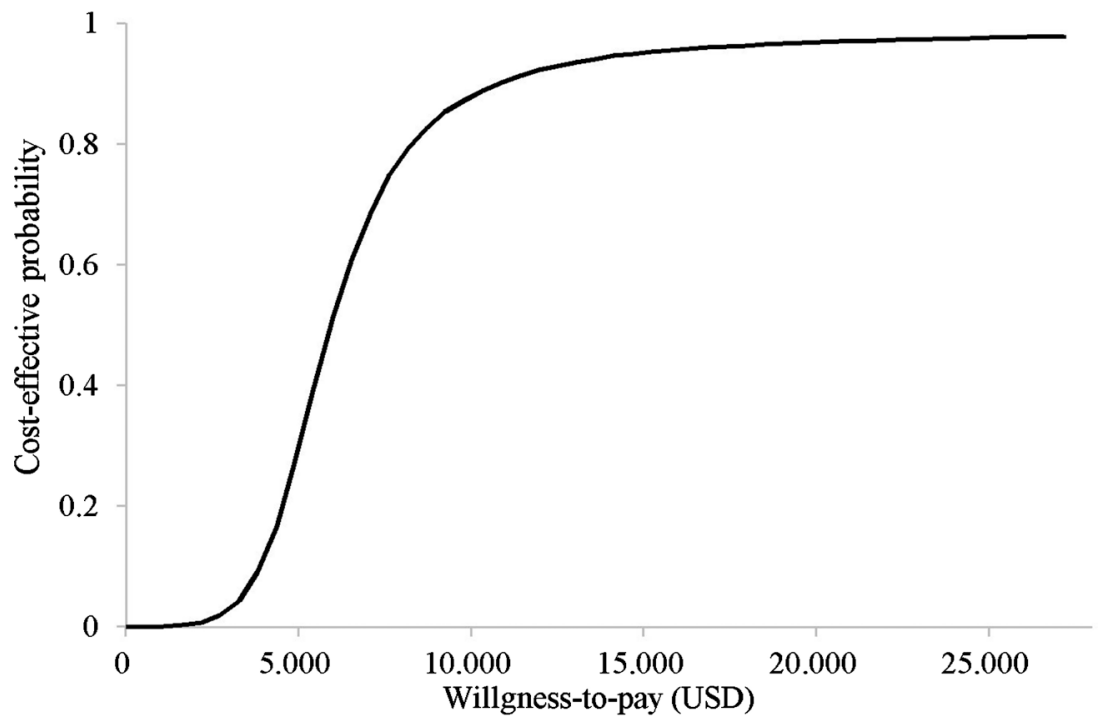

Figure 2. Cost-effectiveness acceptability curve for probabilistic sensitivity analysis of CTZ. At a willingness to pay threshold of 1,200,000 JPY (10,869.6 USD) per QALY. 
neoadjuvant CT for HER2-negative breast cancer. This study was performed to determine whether the cost of health care is favorable according to an economic evaluation that considers cost, treatment efficacy, and quality of life.

The cost was estimated using the direct costs from diagnosis to outcome, including DFS and OS, for patients with breast cancer. Past probabilities and doses of drugs were calculated from JONIE1 Study data without drawing on other studies' results. In this study, we performed base-case analysis using real-world data.

It is essential to distinguish between the different subtypes of breast cancer because CT regimen depends on subtypes. The JONIE1 Study enrolled both HR+ and HR- patients. Therefore, we designed our Markov model on the basis of JONIE1 Study data.

We selected the same utility values in both patient groups because although jaw bone necrosis is a known adverse effect of ZOL, its occurrence is rare, and the NSABP-B35 study reported that anastrozole and tamoxifen did not have significantly different effects on quality of life [15].

The cost-effectiveness analysis was conducted to estimate the associated QALY, ICER, and willingness-to-pay values. At a total cost of 5,699,154 JPY $(51,622.7$ USD), the addition of ZOL to CT for treatment of breast cancer increased the total cost of therapy by 4,988,364 JPY (45,184.5 USD) for the CT alone (ICER: 681,056 JPY per QALY) (6168.99 USD). Over the 10-year time horizon, the CTZ group gained 3.94 QALYs, more than the CT group did (2.9). At a willingness-to-pay threshold of 1,200,000 JPY (10,869.6 USD) per QALY, ZOL was considered to be cost-effective in $90 \%$ of the tested scenarios [16]. This study is subject to the typical limitations of economic models. First, because this study performed cost-effectiveness analysis from health care payers' perspective, it did not include indirect costs. A model including both direct and indirect costs could have different results. Second, a literature review was conducted to obtain health-related utility values in this study because quality of life scores was not collected in the JONIE1 Study. Third, we did not consider the cost of anti-monoclonal agent drugs. However, monoclonal antibodies were used in only a few patients in the JONIE1 Study.

It is convincing that neoadjuvant CTZ for patients with breast cancer would be expected to have statistically significant clinical efficacy. Because a previous study reported that improvement of the pCR rate translated to a higher frequency of survival for 3 years in triple-negative breast cancer [7]. Triple-negative breast cancer has poor prognosis, so it is efficacy to treat CTZ. Another study, ClinicalTrials.gov NCT00242203, reported that ZOL administered with CT may improve DFS and OS in women with ER-stage II/III breast cancer [8]. Also, this study's meta-analysis provides the first data indicating a statistically significant benefit of the addition of ZOL to neoadjuvant CT in terms of pCR in postmenopausal patients with early breast cancer. The addition of ZOL to systemic therapy has shown a survival benefit in postmenopausal patients with low levels of reproductive hormones [17]. 


\section{Conclusion}

ZOL with neoadjuvant CT was associated with QALY improvements compared with neoadjuvant CT alone for HER2-negative breast cancer women.

\section{Acknowledgements}

We thank Richard Lipkin, PhD, from Edanz Group (www.edanzediting.com/ac) for editing a draft of this manuscript.

\section{Conflicts of Interest}

The authors declare no conflicts of interest regarding the publication of this paper.

\section{References}

[1] World Health Organization, International Agency for Research on Cancer. http://globocan.iarc.fr

[2] Cancer Registry and Statistics (2018) Cancer Information Service, National Cancer Center, Japan. https://ganjoho.jp/reg_stat/statistics/stat/summary.html

[3] Yamashita, H., Iwase, H., Toyama, T., Takahashi, S., Sugiura, H., Yoshimoto, N., et al. (2011) Estrogen Receptor-Positive Breast Cancer in Japanese Women: Trends in Incidence, Characteristics, and Prognosis. Annals of Oncology, 22, 1318-1325. https://doi.org/10.1093/annonc/mdq596

[4] Winter, M.C., Holen, I. and Coleman, R.E. (2008) Exploring the Anti-Tumour Activity of Bisphosphonates in Early Breast Cancer. Cancer Treatment Reviews, 34, 453-475. https://doi.org/10.1016/j.ctrv.2008.02.004

[5] Coleman, R., De Boer, R., Eidtmann, H., Llomart, A., Davidson, N., Neven, P., et al. (2013) Zoledronic Acid (Zoledronate) for Postmenopausal Women with Early Breast Cancer Receiving Adjuvant Letrozole (Z0-FAST Study): Final 60-Month Results. Annals of Oncology, 24, 398-405. https://doi.org/10.1093/annonc/mds277

[6] Hasegawa, Y., Tanino, H., Horiguchi, J., Miura, D., Ishikawa, T., Hayashi, M., et al. (2015) Randomized Controlled Trial of Zoledronic Acid Plus Chemotherapy versus Chemotherapy Alone as Neoadjuvant Treatment of HER2-Negative Primary Breast Cancer (JONIE1 Study). PLoS ONE, 10, e0143643. https://doi.org/10.1371/journal.pone.0143643

[7] Ishikawa. T., Akazawa, K., Hasegawa, Y., Tanino, H., Horiguchi, J., Miura, D., et al. (2017) Survival Outcomes of Neoadjuvant Chemotherapy with Zoledronic Acid for HER2-Negative Breast Cancer. Journal of Surgical Research, 220, 46-51. https://doi.org/10.1016/j.jss.2017.05.066

[8] Aft, R.L., Naughton, M., Trinkaus, K. and Weibaecher, K. (2012) Effect of (Neo) Adjuvant Zoledronic Acid on Disease-Free and Overall Survival in Clinical Stage II/III Breast Cancer. British Journal of Cancer, 107, 7-11. https://doi.org/10.1038/bjc.2012.210

[9] Davies, C., Pan, H., Godwin, J., Gray, R., Arriagada, R., Raina, V., et al. (2013) Long-Term Effects of Continuing Adjuvant Tamoxifen to 10 Years versus Stopping at 5 Years after Diagnosis of Estrogen Receptor-Positive Breast Cancer: ATLAS, a Randomized Trial. The Lancet, 381, 805-816. https://doi.org/10.1016/S0140-6736(12)61963-1 
[10] Saito, S., Muneoka, Y., Ishikawa, T. and Akazawa, K. (2017) Cost-Effectiveness of Paclitaxel + Ramucirumab Combination Therapy for Advanced Gastric Cancer Progressing after First-Line Chemotherapy in Japan. Clinical Therapeutics, 39, 2380-2388. https://doi.org/10.1016/j.clinthera.2017.10.017

[11] (2016) National Health Insurance Price List Japan. Jihou Press, Tokyo. (In Japanese)

[12] Freedman, G.M., Li, T., Anderson, P.R., Nicolaou, N. and Konski, A. (2010) Health States of Women Conservative Surgery and Radiation for Breast Cancer. Breast Cancer Research and Treatment, 121, 519-526. https://doi.org/10.1007/s10549-009-0552-5

[13] Shiroiwa, T., Fukuda, T., Shimozuma, K., Mouri, M., Hasegawa, Y., Doihara, H., et al. (2017) Long-Term Health Status as Measured by EQ-5D among Patients with Metastic Breast Cancer: Comparison of First-Line Oral S-1 and Taxane Therapies in the Randomized Phase III SELECT BC Trial. Quality of Life Research, 26, 445-453. https://doi.org/10.1007/s11136-016-1388-1

[14] Central Social Insurance Medical Council Web (2015) Central Social Insurance Medical Council Guideline for Economic Evaluation of Healthcare Technologies for Central Social Insurance Medical Council in Japan. (In Japanese)

https://www.mhlw.go.jp/file/05-Shingikai-12404000-Hokenkyoku-Iryouka/0000104 722.pdf

[15] Ganz, P.A., Cecchini, R.S., Julian, T.B., Margolese, R.G., Costantino, J.O., Vallow, L.A., et al. (2016) Patient-Reported Outcomes with Anastrozole versus Tamoxifen for Postmenopausal Patients with Ductal Carcinoma in Situ Treated with Lumpectomy Plus Radiotherapy (NSABP B-35): A Randomized, Double-Blind, Phase 3 Clinical Trial. The Lancet, 387, 857-865. https://doi.org/10.1016/S0140-6736(15)01169-1

[16] Shiroiwa, T., Igarashi, A., Fukuda, T. and Ikeda, S. (2013) WTP for a QALY and Health States: More Money for Severer Health States? Cost Effectiveness and Resource Allocation, 11, 11-22. https://doi.org/10.1186/1478-7547-11-22 http://www.resource-allocation.com/content/11/1/22

[17] Kroep, J.R., Charehbili, A., Coleman, R.E., Aft, R.L., Hasegawa, Y., Winter, M.C., et al. (2016) Effects of Neoadjuvant Chemotherapy with or without Zoledronic Acid on Pathological Response: A Meta-Analysis of Randomized Trials. European Journal of Cancer, 54, 57-63. https://doi.org/10.1016/j.ejca.2015.10.011 